\title{
COMPUTER METHODS FOR STABILITY ANALYSIS OF THE ROESSER TYPE MODEL OF 2D CONTINUOUS-DISCRETE LINEAR SYSTEMS
}

\author{
MiKoŁAJ BUSŁOWICZ, ANDRZEJ RUSZEWSKI \\ Faculty of Electrical Engineering \\ Białystok University of Technology, ul. Wiejska 45D, 15-351 Białystok, Poland \\ e-mail: \{busmiko, andrusz\}@pb.edu.p1
}

\begin{abstract}
Asymptotic stability of models of 2D continuous-discrete linear systems is considered. Computer methods for investigation of the asymptotic stability of the Roesser type model are given. The methods require computation of eigenvalue-loci of complex matrices or evaluation of complex functions. The effectiveness of the stability tests is demonstrated on numerical examples.
\end{abstract}

Keywords: continuous-discrete system, hybrid system, stability, computer methods.

\section{Introduction}

In continuous-discrete systems both continuous-time and discrete-time components are relevant and interacting and cannot be separated. Such systems are called hybrid systems. Examples of these can be found in the works of Gałkowski et al. (2003), Hespanha (2004), Johanson et al. (2004) and Liberzon (2003). The problems of dynamics and control of hybrid systems were studied by Dymkov (2005), Dymkov et al. (2003; 2004), Gałkowski et al. (2003), Rogers et al., (2007) and Liberzon (2003).

In this paper we consider continuous-discrete linear systems whose models have a structure similar to that of models of 2D discrete-time linear systems. Such models, called 2D continuous-discrete models or 2D hybrid models, were considered by Kaczorek (2002) in the case of positive systems.

A new model of positive 2D hybrid linear systems, similar to the Roesser model of 2D systems, was introduced for standard and for fractional systems by Kaczorek $(2007 ; 2008 \mathrm{a})$. The realization and solvability problems of positive 2D hybrid linear systems were considered by Kaczorek $(2002 ; 2008$ b) as well as Kaczorek et al. (2008) and Sajewski (2009), respectively (see also Kaczorek, 2011, Chapter 12).

The problems of stability and robust stability of 2D continuous-discrete linear systems were investigated by Bistritz (2003; 2004), Xiao (2001), Busłowicz, (2010a; 2010b; 2011a; 2011b) as well as Busłowicz and Ruszewski (2011) (see also Kaczorek, 2011, Chapter 12). The problem of stability of solutions of a class of hybrid difference-difference systems was considered by Marchenko and Loiseau (2009).

The main purpose of this paper is to present computational methods for investigation of asymptotic stability of the Roesser type model of 2D continuous-discrete linear systems.

The following notation will be used: $\mathbb{R}$ is the set of real numbers, $\mathbb{R}_{+}=[0, \infty], \mathbb{Z}_{+}$is the set of non-negative integers, $\mathbb{R}^{n \times m}$ is the set of real $n \times m$ matrices, $\lambda_{i}\{X\}$ is the $i$-th eigenvalue of matrix $X$.

\section{Problem formulation}

Consider the state equation of the Roesser type model of $2 \mathrm{D}$ continuous-discrete linear system (for $i \in \mathbb{Z}_{+}$and $\left.t \in \mathbb{R}_{+}\right)$

$$
\begin{aligned}
\dot{x}_{1}(t, i) & =A_{11} x_{1}(t, i)+A_{12} x_{2}(t, i)+B_{1} u(t, i), \\
x_{2}(t, i+1) & =A_{21} x_{1}(t, i)+A_{22} x_{2}(t, i)+B_{2} u(t, i),
\end{aligned}
$$

where

$$
\begin{array}{ll}
\dot{x}_{1}(t, i)=\partial x_{1}(t, i) / \partial t, & x_{1}(t, i) \in \mathbb{R}^{n_{1}}, \\
x_{2}(t, i) \in \mathbb{R}^{n_{2}}, & u(t, i) \in \mathbb{R}^{m}
\end{array}
$$


and

$$
\begin{aligned}
A_{11} & \in \mathbb{R}^{n_{1} \times n_{1}}, & A_{12} & \in \mathbb{R}^{n_{1} \times n_{2}}, \\
A_{21} & \in \mathbb{R}^{n_{2} \times n_{1}}, & A_{22} & \in \mathbb{R}^{n_{2} \times n_{2}}, \\
B_{1} & \in \mathbb{R}^{n_{1} \times m}, & B_{2} & \in \mathbb{R}^{n_{2} \times m} .
\end{aligned}
$$

The model (1) was introduced by Kaczorek (2007). A similar model was also considered by Dymkov et al. (2003).

The boundary conditions for the model (1) are as follows:

$$
\begin{array}{ll}
x_{1}(0, i)=x_{1}(i), & i \in \mathbb{Z}_{+}, \\
x_{2}(t, 0)=x_{2}(t), & t \in \mathbb{R}_{+} .
\end{array}
$$

The model (1) can be written in the form

$$
\begin{aligned}
{\left[\begin{array}{l}
\dot{x}_{1}(t, i) \\
x_{2}(t, i+1)
\end{array}\right]=} & {\left[\begin{array}{ll}
A_{11} & A_{12} \\
A_{21} & A_{22}
\end{array}\right]\left[\begin{array}{l}
x_{1}(t, i) \\
x_{2}(t, i)
\end{array}\right] } \\
& +\left[\begin{array}{l}
B_{1} \\
B_{2}
\end{array}\right] u(t, i) .
\end{aligned}
$$

From (3) it follows that the model (1) has a structure similar to that of the Roesser type model (Kaczorek, 2002). The characteristic matrix of the model (1) has the form

$$
H(s, z)=\operatorname{det}\left[\begin{array}{ll}
s I_{n_{1}}-A_{11} & -A_{12} \\
-A_{21} & z I_{n_{2}}-A_{22}
\end{array}\right],
$$

where $s$ and $z$ are complex variables.

The characteristic function of the model (1) is a polynomial in two independent variables $s$ and $z$ of the form

$$
\begin{aligned}
w(s, z) & =\operatorname{det} H(s, z) \\
& =\sum_{k=0}^{n_{1}} \sum_{j=0}^{n_{2}} a_{k j} s^{k} z^{j}, \quad a_{n_{1} n_{2}}=1 .
\end{aligned}
$$

Definition 1. The model (1) is called asymptotically stable (or Hurwitz-Schur stable) if for $u(t, i) \equiv 0$ and the bounded boundary conditions (2) the condition $x(t, i) \rightarrow 0$ holds as $t, i \rightarrow \infty$.

Following Bistritz $(2003 ; 2004)$ as well as Guiver and Bose (1981), we have the following theorem.

Theorem 1. The model (1) is asymptotically stable if and only if

$$
w(s, z) \neq 0, \quad \operatorname{Re} s \geq 0, \quad|z| \geq 1 .
$$

The polynomial (5) satisfying the condition (6) is called continuous-discrete stable (C-D stable) or HurwitzSchur stable. Several algebraic methods for asymptotic stability checking of such bivariate polynomials were given by Bistritz (2003; 2004) and Xiao (2001).
Computational methods for investigation of asymptotic stability of special classes of the FornasiniMarchesini and the Roesser type models of continuousdiscrete linear systems was given by Busłowicz (2011a). These methods require computation of the eigenvalue-loci of complex matrices.

Analytical conditions for asymptotic stability and for robust stability of the general scalar model and the scalar model (1) of continuous-discrete linear systems were derived by Busłowicz (2010b; 2011b; 2010a).

The main purpose of this paper is to present computational methods for checking the condition (6) of the asymptotic stability of the continuous-discrete linear model (1) which does not require a priori knowledge of the characteristic bivariate polynomial (5).

\section{Solution of the problem}

Theorem 2. The model (1) is asymptotically stable if and only if the following two conditions hold:

$$
\begin{gathered}
w\left(s, e^{j \omega}\right) \neq 0, \quad \operatorname{Re} s \geq 0, \\
\forall \omega \in \Omega=[0,2 \pi], \quad j^{2}=-1, \\
w(j y, z) \neq 0, \quad|z| \geq 1, \\
\forall y \in[0, \infty), \quad j^{2}=-1 .
\end{gathered}
$$

Proof. From the work of Guiver and Bose (1981) it follows that (6) is equivalent to the conditions

$$
\begin{aligned}
& w(s, z) \neq 0, \quad \operatorname{Re} s \geq 0, \quad|z|=1, \\
& w(s, z) \neq 0, \quad \operatorname{Re} s=0, \quad|z| \geq 1 .
\end{aligned}
$$

It is easy to see that (9) and (10) can be written in the forms (7) and (8), respectively.

Lemma 1. If the model (11) is asymptotically stable, then

$$
\operatorname{Re} \lambda_{l}\left(A_{11}\right)<0, \quad l=1,2, \ldots, n_{1}
$$

and

$$
\left|\lambda_{i}\left(A_{22}\right)\right|<1, \quad i=1,2, \ldots, n_{2} .
$$

Proof. From the first equation of (1) for $A_{12} \equiv 0$ and $B_{1} \equiv 0$ we obtain the homogeneous state equation of the continuous-time linear system

$$
\dot{x}_{1}(t, i)=A_{11} x_{1}(t, i) .
$$

The system (13) is asymptotically stable if and only if the condition (11) holds, i.e., the matrix $A_{11}$ is Hurwitz stable (e.g., it is a Hurwitz matrix).

Similarly, substitution of $A_{21} \equiv 0$ and $B_{2} \equiv 0$ in the second equation of (1) yields the homogeneous state equation of discrete-time linear system

$$
x_{2}(t, i+1)=A_{22} x_{2}(t, i),
$$


which is asymptotically stable if and only if the condition (12) holds, i.e., the matrix $A_{22}$ is Schur stable (is a Schur matrix).

If the model (1) is asymptotically stable, then, in particular, it is asymptotically stable for $A_{12} \equiv 0$ or $A_{21} \equiv 0$. Hence, the conditions (11) and (12) are necessary for the asymptotic stability of the model (1).

To show that the conditions (11) and (12) are not sufficient, we consider the scalar system (1) with $A_{11}=-1$, $A_{22}=0$ (11) and (12) hold) and $A_{12} A_{21}=1$. In this case the characteristic equation has the form $s z+z-1=0$. From this equation we have that if, for example, $z=1 / 2$, then $s=1>0$, and if $s=0$, then $z=1$. This means that there exist values of zeroes of the characteristic equation which do not satisfy the condition (6) and the system is unstable.

Using the rules for computing the determinant of block matrices (Kaczorek, 1998), we obtain that the characteristic matrix (4) of the model (1) can be computed from one of the following equivalent formulae:

$$
\begin{aligned}
& H(s, z)=\left[z I_{n_{2}}-A_{22}\right]\left[s I_{n_{1}}-S_{1}(z)\right], \\
& H(s, z)=\left[s I_{n_{1}}-A_{11}\right]\left[z I_{n_{2}}-S_{2}(s)\right],
\end{aligned}
$$

where

$$
\begin{aligned}
& S_{1}(z)=A_{11}+A_{12}\left(z I_{n_{2}}-A_{22}\right)^{-1} A_{21}, \\
& S_{2}(s)=A_{22}+A_{21}\left(s I_{n_{1}}-A_{11}\right)^{-1} A_{12} .
\end{aligned}
$$

Using (5) and (15), 16) we can write

$$
\begin{aligned}
& w(s, z)=\operatorname{det}\left[z I_{n_{2}}-A_{22}\right] \operatorname{det}\left[s I_{n_{1}}-S_{1}(z)\right], \\
& w(s, z)=\operatorname{det}\left[s I_{n_{1}}-A_{11}\right] \operatorname{det}\left[z I_{n_{2}}-S_{2}(s)\right] .
\end{aligned}
$$

From (15) for $z=e^{j \omega}$ we have

$$
H\left(s, e^{j \omega}\right)=\left[I_{n_{2}} e^{j \omega}-A_{22}\right]\left[s I_{n_{1}}-S_{1}\left(e^{j \omega}\right)\right],
$$

where

$$
S_{1}\left(e^{j \omega}\right)=A_{11}+A_{12}\left(I_{n_{2}} e^{j \omega}-A_{22}\right)^{-1} A_{21} .
$$

Lemma 2. Let the necessary condition (12) be satisfied. The condition (7) holds if and only if all eigenvalues of the complex matrix (22) have negative real parts for all $\omega \in[0,2 \pi]$.

Proof. From (21) we have

$$
w\left(s, e^{j \omega}\right)=\operatorname{det}\left[I_{n_{2}} e^{j \omega}-A_{22}\right] \operatorname{det}\left[s I_{n_{1}}-S_{1}\left(e^{j \omega}\right)\right] .
$$

If (12) holds, then the matrix $I_{n_{2}} e^{j \omega}-A_{22}$ is nonsingular for all $\omega \in \Omega$. Hence, from (23) it follows that the condition (7) is satisfied if and only if

$$
\operatorname{det}\left[s I_{n_{1}}-S_{1}\left(e^{j \omega}\right)\right] \neq 0, \quad \operatorname{Re} s \geq 0, \quad \forall \omega \in \Omega .
$$

Satisfaction of (24) means that all eigenvalues of the complex matrix 22 have negative real parts for all $\omega \in[0,2 \pi]$.

From (16) for $s=j y$ we have

$$
H(j y, z)=\left[j y I_{n_{1}}-A_{11}\right]\left[z I_{n_{2}}-S_{2}(j y)\right]
$$

and

$$
w(j y, z)=\operatorname{det}\left[j y I_{n_{1}}-A_{11}\right] \operatorname{det}\left[z I_{n_{2}}-S_{2}(j y)\right],
$$

where

$$
S_{2}(j y)=A_{22}+A_{21}\left(j y I_{n_{1}}-A_{11}\right)^{-1} A_{12} .
$$

Lemma 3. Let the necessary condition (11) be satisfied. The condition (8) holds if and only if all eigenvalues of the complex matrix (27) have absolute values less than one for all $y \geq 0$.

Proof. If (11) holds, then the matrix $j y I_{n_{1}}-A_{11}$ is nonsingular for all $y \geq 0$. From (26) we have that the condition (8) is satisfied if and only if

$$
\operatorname{det}\left[z I_{n_{2}}-S_{2}(j y)\right] \neq 0, \quad|z| \geq 1, \quad \forall y \in[0, \infty),
$$

i.e., all eigenvalues of the matrix (27) have absolute values less than one for all $y \geq 0$.

The conditions of Lemmas 2 and 3 can be written in the following forms:

$$
\operatorname{Re} \lambda_{i}\left\{S_{1}\left(e^{j \omega}\right)\right\}<0, \quad \forall \omega \in \Omega, \quad i=1,2, \ldots, n_{1},
$$

and

$$
\left|\lambda_{i}\left\{S_{2}(j y)\right\}\right|<1, \quad \forall y \geq 0, \quad i=1,2, \ldots, n_{2},
$$

respectively.

Theorem 3. The model (1) is asymptotically stable if and only if the conditions (11), (12), (29) and (30) are satisfied.

Proof. The proof follows directly from Theorem 2 and Lemmas 1-3.

Busłowicz (2011a) showed that, if $A_{11} \neq I_{n_{1}}$ and $A_{22} \neq \pm I_{n_{2}}$, then the the model (1) is asymptotically stable if and only if the conditions (29) and (30) hold. This means that the result of Busłowicz (2011a) concerns only a special case of the model (1). Moreover, if $A_{11} \neq I_{n_{1}}, A_{22} \neq \pm I_{n_{2}}$ and the necessary conditions (11), (12) are not satisfied, then applying the result of $\mathrm{Bu}$ słowicz (2011a) we have to check the conditions 29) and (30), whereas applying Theorem 3 we simply conclude that the model (1) is not asymptotically stable.

Xiao (2001) showed that the Roesser model of 2D continuous-discrete systems is asymptotically stable if and only if $A_{11}$ is a Hurwitz stable matrix and the matrix

$$
A_{22}+A_{21}\left(s I_{n_{1}}-A_{11}\right)^{-1} A_{12}
$$


is Schur stable for $\operatorname{Re} s=0$.

Comparison of the above and Theorem 3 gives that the result of Xiao (2001) is equivalent to the necessary condition (11) and the condition (30), while satisfaction of (12) and 29) is unnecessary. Below, by a counterexample, we show that the result of Xiao (2001) is incorrect.

Consider the scalar system (1) with $A_{11}=-1, A_{22}=0$ and $A_{12} A_{21}=0.5$. It is easy to see that $A_{11}$ is Hurwitz stable and

$$
A_{22}+A_{21}\left(s I_{n_{1}}-A_{11}\right)^{-1} A_{12}=\frac{0.5}{s+1}
$$

is Schur stable for $\operatorname{Re} s=0$. This means that the conditions of Xiao (2011) are satisfied.

The zeroes of the characteristic function $w(s, z)=s z+z-0.5$ of the system satisfy the relationship $s=-1+1 /(2 z)$. For example, if $z=0.25$, then $s=1$. This means that the condition (6) is not satisfied and the system is unstable.

From the above it follows that the main result of Xiao (2001) is incorrect.

Example 1. Consider the model (1) with the matrices

$$
\begin{array}{lll}
A_{11} & =\left[\begin{array}{ll}
-3 & 1 \\
0.1 & -1
\end{array}\right], & A_{12}=\left[\begin{array}{ll}
1.5 & -1 \\
-1 & 0
\end{array}\right], \\
A_{21}=\left[\begin{array}{ll}
0.3 & 0.1 \\
2 & 1
\end{array}\right], & A_{22}=\left[\begin{array}{ll}
0.5 & 0 \\
1 & 0.2
\end{array}\right] .
\end{array}
$$

Computing eigenvalues of $A_{11}$ and $A_{22}$, we obtain $s_{1}=-0.951, s_{2}=-3.049$ and $z_{1}=0.2, z_{2}=0.5$, respectively. This means that the necessary conditions (11) and (12) hold, i.e., the matrix $A_{11}$ is Hurwitz stable and the matrix $A_{22}$ is Schur stable.

The eigenvalues of the matrix $S_{1}\left(e^{j \omega}\right)$ for $\omega \in \Omega$ and the matrix $S_{2}(j y)$ for $y \in[-100,100]$ are shown in Figs. 1 and 2. If is easy to check that eigenvalues of $S_{2}(j y)$ remain in the unit circle for all $y$ with $|y|>100$.

From Figs. 1 and 2 it follows that the conditions (29) and (30) of Theorem 3 are satisfied and the model is asymptotically stable.

The above methods for checking the conditions of Theorem 3 may be inconvenient with respect to computational problems, particularly in the case of ill conditioned matrices.

Therefore, now we present a new method for investigation of the asymptotic stability of the model (1) which does not require computation of eigenvalues of the complex matrices (22) and (27). In this method, computation of determinants of some matrices is necessary.

Consider the polynomial

$$
w_{1}\left(s, e^{j \omega}\right)=\operatorname{det}\left(s I_{n_{1}}-S_{1}\left(e^{j \omega}\right)\right),
$$

where the matrix $S_{1}\left(e^{j \omega}\right)$ is defined by (22). From the classical Mikhailov theorem (see, e.g., Busłowicz, 1997;

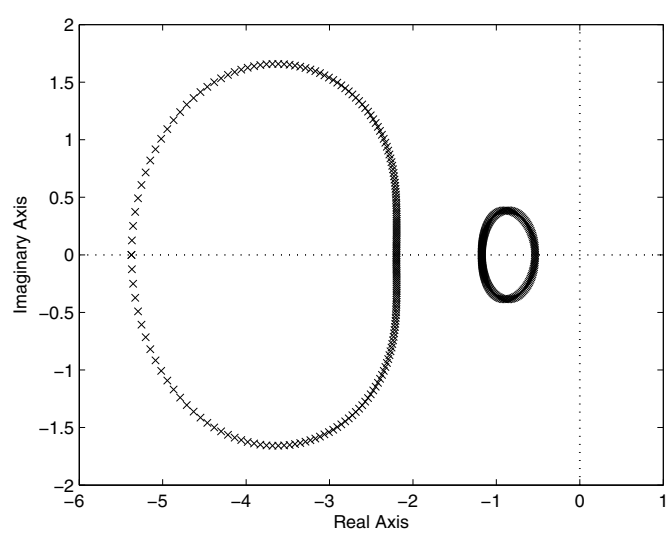

Fig. 1. Eigenvalues of $S_{1}\left(e^{j \omega}\right), \omega \in[0,2 \pi]$.

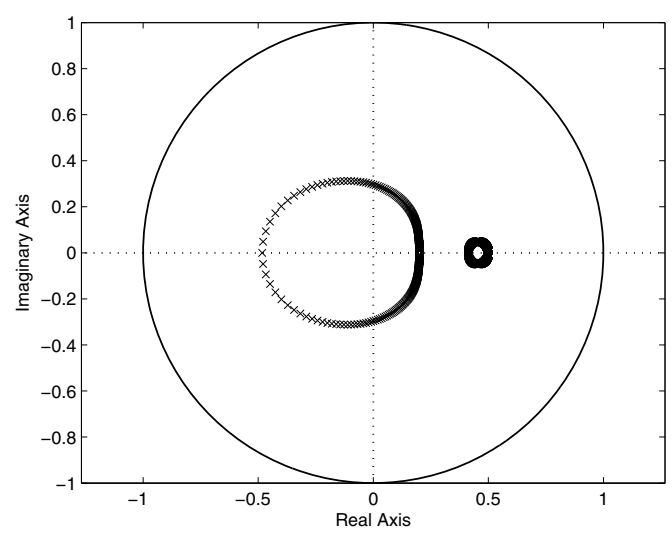

Fig. 2. Eigenvalues of $S_{2}(j y), y \in[-100,100]$.

Keel and Bhattacharyya, 2000), it follows that the condition (24) holds if and only if for any fixed $\omega \in[0,2 \pi]$ the plot of $w_{1}\left(j y, e^{j \omega}\right)$ starts for $y=0$ in the point $w_{1}\left(0, e^{j \omega}\right)=\operatorname{det}\left(-S_{1}\left(e^{j \omega}\right)\right)$ and runs in the positive direction by $n_{1}$ quadrants of the complex plane (missing the origin of this plane) if $y$ increases from 0 to $+\infty$.

It is easy to see that the plot of $w_{1}\left(j y, e^{j \omega}\right)$ quickly tends to infinity as $y$ grows to $\infty$. Therefore, direct application of the Mikhailov theorem to checking the condition (24) is not practically reliable.

To remove this difficulty, we introduce the rational function

$$
\varphi_{1}\left(j y, e^{j \omega}\right)=\frac{w_{1}\left(j y, e^{j \omega}\right)}{w_{1 o}(j y)}, \quad \omega \in \Omega,
$$

instead of $w_{1}\left(j y, e^{j \omega}\right)$, where $w_{1 o}(s)$ is any Hurwitz stable polynomial of degree $n_{1}$.

Lemma 4. The condition (24) holds if and only if for all fixed $y \geq 0$ the plot of the function (33) does not encircle or cross the origin of the complex plane. 
Proof. If the reference polynomial $w_{1 o}(s)$ is Hurwitz stable, then from the argument principle we have

$$
\Delta \arg _{y \in(-\infty, \infty)} w_{1 o}(j y)=n_{1} \pi .
$$

From (33) it follows that, for any fixed $\omega \in \Omega$,

$\Delta \arg \varphi_{1}\left(j y, e^{j \omega}\right)=\Delta \arg w_{1}\left(j y, e^{j \omega}\right)-\Delta \arg w_{1 o}(j y)$.

The matrix (22) for any fixed $\omega \in \Omega$ is Hurwitz stable if and only if

$$
\begin{aligned}
& \Delta \arg _{y \in(-\infty, \infty)} w_{1}\left(j y, e^{j \omega}\right) \\
& \quad=\Delta \arg _{y \in(-\infty, \infty)} w_{1 o}(j y)=n_{1} \pi,
\end{aligned}
$$

which holds if and only if $\Delta \arg \varphi_{1}\left(j y, e^{j \omega}\right)=0$, i.e., the plot of 33 does not encircle or cross the origin of the complex plane for all fixed $y \geq 0$.

Taking into account all $\omega \in \Omega$, we obtain that the above holds $\forall \omega \in \Omega$ if and only if for all fixed $y \geq 0$ the plot of (33) as a function of $\omega \in \Omega$ does not encircle or cross the origin of the complex plane.

The reference polynomial $w_{1 o}(s)$ can be chosen in the form

$$
w_{1}(s, 1)=\operatorname{det}\left(s I_{n_{1}}-S_{1}(1)\right),
$$

where $S_{1}(1)=A_{11}+A_{12}\left(I_{n_{2}}-A_{22}\right)^{-1} A_{21}$, which we get from (32) and (22) by substituting $\omega=0$. Hurwitz stability of (36) is necessary for Hurwitz stability of the complex polynomial (32) for all $\omega \in \Omega$.

If $w_{1 o}(s)=w_{1}(s, 1)$, then

$$
\varphi_{1}\left(j y, e^{j \omega}\right)=\frac{w_{1}\left(j y, e^{j \omega}\right)}{w_{1}(j y, 1)}, \quad \omega \in \Omega .
$$

The plot of (37) as a function of $\omega \in \Omega$ (with any fixed $y \geq 0$ ) is a closed curve. It begins with $\omega=0$ and ends with $\omega=2 \pi$ in the point $\varphi_{1}(j y, 1)=1$. It is easy to check that, as $y \rightarrow \infty$, the closed curve (37) reduces to the point $(1, j 0)$.

Now, we consider the complex polynomial

$$
w_{2}(j y, z)=\operatorname{det}\left(z I_{n_{2}}-S_{2}(j y)\right),
$$

where the matrix $S_{2}(j y)$ is defined by (27). Let $w_{2 o}(z)$ be any Schur stable polynomial of degree $n_{2}$.

Proceeding similarly as in the case of Lemma 4, we obtain the following lemma.

Lemma 5. The condition (28) holds if and only if for all fixed $y \geq 0$ the plot of the function

$$
\varphi_{2}\left(j y, e^{j \omega}\right)=\frac{w_{2}\left(j y, e^{j \omega}\right)}{w_{2 o}\left(e^{j \omega}\right)}, \quad \omega \in \Omega,
$$

does not encircle or cross the origin of the complex plane, where $w_{2}\left(j y, e^{j \omega}\right)$ has the form (38) for $z=e^{j \omega}$.
The reference polynomial $w_{2 o}(z)$ can be chosen in the form

$$
w_{2}(0, z)=\operatorname{det}\left(z I_{n_{2}}-S_{2}(0)\right),
$$

where $S_{2}(0)=A_{22}+A_{21}\left(-A_{11}\right)^{-1} A_{12}$. Schur stability of (40) is necessary for Schur stability of the complex polynomial (38) for all $y \geq 0$.

If $w_{2 o}(z)=w_{2}(0, z)$, then

$$
\varphi_{2}\left(j y, e^{j \omega}\right)=\frac{w_{2}\left(j y, e^{j \omega}\right)}{w_{2}\left(0, e^{j \omega}\right)}, \quad \omega \in \Omega .
$$

The plot of (41) as a function of $\omega \in \Omega$ with the fixed $y \geq 0$ is a closed curve. It begins with $\omega=0$ and ends with $\omega=2 \pi$ at the point

$$
\varphi_{2}(j y, 1)=\frac{w_{2}(j y, 1)}{w_{2}(0,1)}=\frac{\operatorname{det}\left(I_{n_{2}}-S_{2}(j y)\right)}{\operatorname{det}\left(I_{n_{2}}-S_{2}(0)\right)} .
$$

It is easy to see that $\varphi_{2}(0,1)=1$.

From 27) it follows that

$$
S_{2}(\infty)=\lim _{y \rightarrow \infty} S_{2}(j y)=A_{22} .
$$

Hence, from (38) and (41) we have

$$
\begin{aligned}
\varphi_{2}\left(\infty, e^{j \omega}\right) & =\lim _{y \rightarrow \infty} \varphi_{2}\left(j y, e^{j \omega}\right) \\
& =\frac{\operatorname{det}\left(e^{j \omega} I_{n_{2}}-A_{22}\right)}{\operatorname{det}\left(e^{j \omega} I_{n_{2}}-S_{2}(0)\right)}, \quad \omega \in \Omega .
\end{aligned}
$$

From the above it follows that, as $y \rightarrow \infty$, the plot of (41) tends to the closed curve (44) with endpoints (for $\omega=0$ and $\omega=2 \pi)$,

$$
\varphi_{2}(\infty, 1)=\frac{\operatorname{det}\left(I_{n_{2}}-A_{22}\right)}{\operatorname{det}\left(I_{n_{2}}-S_{2}(0)\right)} .
$$

From Theorem 3 as well as Lemmas 4 and 5 we have the following result.

Theorem 4. Assume that the necessary conditions (11) and (12) are satisfied. The model (1) is asymptotically stable if and only if the following two conditions hold:

(i) plots of the function (37) do not encircle or cross the origin of the complex plane for all fixed $y \geq 0$;

(ii) plots of the function (41) do not encircle or cross the origin of the complex plane for all fixed $y \geq 0$.

Applying the computational method given in Theorem 4, we can formulate the following remark.

Remark 1. The range $Y=\left[0, y_{f}\right]$ of the values of the parameter $y$ should be suitably large, so that from plots of the functions (37) and 411 for $y \in Y$ we could ascertain the fulfilment of the conditions of Theorem 4 for all $y \geq 0$. For any fixed $y \in Y$, determined with an appropriately small step $\Delta y$, plots of the functions (37) and (41) should be drawn separately to discretize the range $\Omega=[0,2 \pi]$ with a sufficiently small step $\Delta \omega$. 


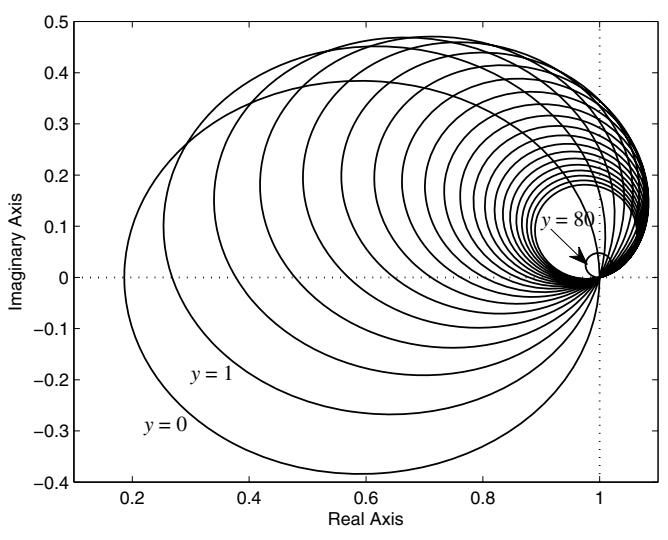

Fig. 3. Plots of 37 for $y=0,1, \ldots, 10$ and $y=80$.

Example 2. Using Theorem 4, we check the asymptotic stability of the model (1) with the matrices (31).

In Example 1 it was shown that the necessary conditions (11) and (12) hold.

Computing from (36) and (40) the reference polynomials, we obtain, respectively,

$$
\begin{aligned}
w_{1}(s, 1) & =s^{2}+6.55 s+6.32 \\
& =(s+1.176)(s+5.374)
\end{aligned}
$$

and

$$
\begin{aligned}
w_{2}(0, z) & =z^{2}+0.0707 z-0.199 \\
& =(z+0.4121)(z-0.4828) .
\end{aligned}
$$

It follows that the reference polynomial 46) is Hurwitz stable and the reference polynomial (47) is Schur stable.

Plots of 377 for $y=0,1, \ldots, 10$ and for $y=80$ are shown in Fig. 3. Figure 4 shows plots of (41) for $y=0,1, \ldots, 10$ and for $y=\infty$ (computed from 444). The range $\Omega=[0,2 \pi]$ for all plots was discretized with the step $\Delta \omega=0.01 \pi$.

From Figs. 3 and 4 it follows that the plots do not encircle the origin of the complex plane for all $y \geq 0$. According to Theorem 4, this means that the model (1), (31) is Hurwitz-Schur stable.

\section{Concluding remarks}

Simple necessary conditions and computational methods for investigation of asymptotic stability of the Roesser type model 11 of 2D continuous-discrete linear systems have been given in Lemma 1 and in Theorems 3 and 4, respectively. The first method requires computation of the eigenvalue-loci of complex matrices (22) and (27). The second method requires evaluation of functions (37) and (41). This method is simpler from the computational point of view.

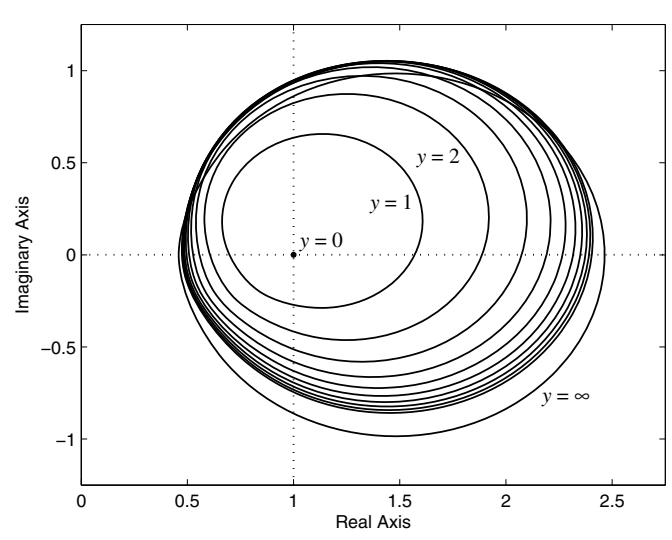

Fig. 4. Plot of 411 for $y=0,1, \ldots, 10$ and $y=\infty$.

The method of Theorem 4 was applied by Busłowicz and Ruszewski (2011) to asymptotic stability analysis of the first Fornasini-Marchesini type model.

It has been also shown that the main result of Xiao (2001) is incorrect.

\section{Acknowledgment}

The authors wish to thanks the anonymous reviewers for their valuable comments and suggestions.

This work was supported by the Ministry of Science and Higher Education of Poland under the grant no. S/WE/1/2011.

\section{References}

Bistritz, Y. (2003). A stability test for continuous-discrete bivariate polynomials, Proceedings of the 2003 IEEE International Symposium on Circuits and Systems, Bangkok, Thailand, Vol. 3, pp. 682-685.

Bistritz, Y. (2004). Immittance and telepolation-based procedures to test stability of continuous-discrete bivariate polynomials, Proceedings of the 2004 IEEE International Symposium on Circuits and Systems, Vancouver, Canada, Vol. 3, pp. 293-296.

Busłowicz, M. (1997). Stability of Linear Time-invariant Systems with Uncertain Parameters, Technical University of Białystok, Białystok, (in Polish).

Busłowicz, M. (2010a). Robust stability of the new general 2D model of a class of continuous-discrete linear systems, Bulletin of the Polish Academy of Sciences: Technical Sciences 57(4): 561-565.

Busłowicz, M. (2010b). Stability and robust stability conditions for general model of scalar continuous-discrete linear systems, Pomiary, Automatyka, Kontrola 56(2): 133-135.

Busłowicz, M. (2011a). Computational methods for investigation of stability of models of 2D continuous-discrete linear systems, Journal of Automation, Mobile Robotics and Intelligent Systems 5(1): 3-7. 
Busłowicz, M. (2011b). Improved stability and robust stability conditions for general model of scalar continuous-discrete linear systems, Pomiary, Automatyka, Kontrola 57(2): 188189.

Busłowicz, M. and Ruszewski, A. (2011). Stability investigation of continuous-discrete linear systems, Pomiary, Automatyka, Robotyka 2(2): 566-575, (on CD-ROM, in Polish).

Dymkov, M. (2005). Extremal Problems in Multiparameter Control Systems, BGEU Press, Minsk, (in Russian).

Dymkov, M., Gaishun, I., Rogers, E., Gałkowski, K. and Owens, D.H. (2004). Control theory for a class of 2D continuousdiscrete linear systems, International Journal of Control 77(9): 847-860.

Dymkov M., Rogers E., Dymkou S., Gałkowski, K. and Owens D.H. (2003). Delay system approach to linear differential repetitive processes, Proceedings of the IFAC Workshop on Time-Delay Systems (TDS 2003), Rocquencourt, France, (CD-ROM).

Gałkowski, K., Rogers, E., Paszke, W. and Owens, D.H. (2003). Linear repetitive process control theory applied to a physical example, International Journal of Applied Mathematics and Computer Science 13(1): 87-99.

Guiver, J.P. and Bose, N.K. (1981). On test for zero-sets of multivariate polynomials in noncompact polynomials, Proceedings of the IEEE 69(4): 467-469.

Hespanha, J. (2004). Stochastic hybrid systems: Application to communication networks, Technical report, Department of Electrical and Computer Engineering, University of California, Santa Barbara, CA.

Johanson, K., Lygeros, J. and Sastry, S. (2004). Modelling hybrid systems, in H. Unbehauen (Ed.), Encyclopedia of Life Support Systems, EOLSS, Berlin.

Kaczorek, T. (1998). Vectors and Matrices in Automatics and Electrotechnics, WNT, Warsaw, p. 70, (in Polish).

Kaczorek, T. (2002). Positive $1 D$ and $2 D$ Systems, SpringerVerlag, London.

Kaczorek, T. (2007). Positive 2D hybrid linear systems, Bulletin of the Polish Academy of Sciences: Technical Sciences 55(4): 351-358.

Kaczorek, T. (2008a). Positive fractional 2D hybrid linear systems, Bulletin of the Polish Academy of Sciences: Technical Sciences 56(3): 273-277.

Kaczorek, T. (2008b). Realization problem for positive 2D hybrid systems, International Journal for Computation and Mathematics in Electrical and Electronic Engineering, COMPEL 27(3): 613-623.

Kaczorek, T. (2011). Selected Problems of Fractional Systems Theory, Lecture Notes in Control and Information Sciences, Vol. 411, Springer-Verlag, Berlin.

Kaczorek, T., Marchenko, V. and Sajewski, Ł. (2008). Solvability of 2D hybrid linear systems - Comparison of the different methods, Acta Mechanica et Automatica 2(2): 59-66.
Keel, L.H. and Bhattacharyya, S.P. (2000). A generalization of Mikhailov's criterion with applications, Proceedings of the American Control Conference, Chicago, IL, USA, Vol. 6, pp. 4311-4315.

Liberzon, D. (2003). Switching in Systems and Control, Birkhauser, Boston, MA.

Sajewski, Ł. (2009). Solution of 2D singular hybrid linear systems, Kybernetes 38(7/8): 1079-1092.

Marchenko V.M. and Loiseau J.J. (2009). On the stability of hybrid difference-differential systems, Differential Equation 45(5), 743-756.

Rogers, E., Gałkowski, K. and Owens, D.H. (2007). Control Systems Theory and Applications for Linear Repetitive Processes, Lecture Notes in Control and Information Sciences, Vol. 349 , Springer-Verlag, Berlin.

Xiao, Y. (2001). Stability test for 2-D continuous-discrete systems. Proceedings of the 40th IEEE Conference on Decision and Control, Orlando, FL, USA, Vol. 4, pp. 3649-3654.

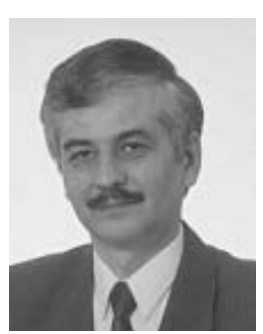

Mikołaj Busłowicz received the M.Sc., Ph.D and D.Sc. degrees in electrical engineering in 1974, 1977 and 1988, respectively, all from the Faculty of Electrical Engineering of the Warsaw University of Technology. Since 1978 he has been working at the Białystok University of Technology. In the years 1988-1993 and 1993-1999 he was respectively a deputy dean and the dean of the Faculty of Electrical Engineering, and in the period of 1999-2005 he was a deputy rector of the Białystok University of Technology. In 2002 he became a professor and since 2005 has been a full professor at the university. Currently, he is the head of the Department of Automatic Control and Electronics. Since 2004 he has been a member of the Committee on Automatic Control and Robotics of the Polish Academy of Sciences. His main research interests include the analysis and synthesis of time delay systems, positive systems, fractional systems and continuous-discrete systems. He has published 3 books and about 170 scientific papers.

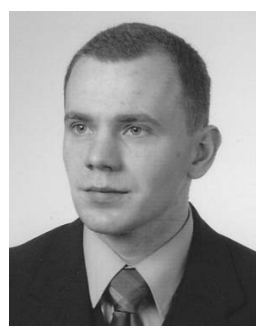

Andrzej Ruszewski graduated from the Faculty of Electrical Engineering of the Białystok University of Technology. He has been working at the Faculty of Electrical Engineering since 2000. Currently he is an assistant professor in the Department of Automatic Control and Electronics. He received his doctoral degree in electrical engineering in 2008. He is an author and co-author of over 30 scientific papers. His research interests include the analysis and synthesis of control systems, particularly systems with time delay and fractional systems.

Received: 25 February 2011 Revised: 11 June 2011 Re-revised: 16 October 2011 